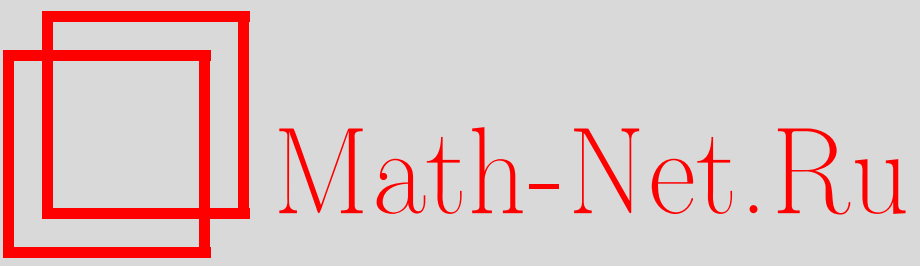

А. В. Зотов, А. М. Левин, Интегрируемая система взаимодействующих эллиптических волчков, ТМФ, 2006, том 146, номер 1, 55-64

DOI: https://doi.org/10.4213/tmf2008

Использование Общероссийского математического портала Math-Net.Ru подразумевает, что вы прочитали и согласны с пользовательским соглашением

http://www.mathnet.ru/rus/agreement

Параметры загрузки:

IP : 35.173 .137 .237

26 апреля 2023 г., 16:19:47 
ТЕОРЕТИЧЕСКАЯ

И МАТЕМАТИЧЕСКАЯ

ФИЗИКА

Том 146, № 1

январь, 2006

(C) 2006 г.

А. В. Зотов* , А. М. Левин ${ }^{\dagger}$

\section{ИНТЕГРИРУЕМАЯ СИСТЕМА ВЗАИМОДЕЙСТВУЮЩИХ ЭЛЛИПТИЧЕСКИХ ВОЛЧКОВ}

Предложен способ построения системы взаимодействующих эллиптических волчков. Полученная система интегрируема по построению и симплектоморфна эллиптической системе Калоджеро-Мозера.

Ключевые слова: интегрируемые системы, алгебраическая геометрия, симплектическая геометрия.

\section{1. СИСТЕМЫ ГОДЕНА НА ЭЛЛИПТИЧЕСКОЙ КРИВОЙ}

Пусть $\Sigma_{\tau}$ - эллиптическая кривая с периодами $(1, \tau),\left\{z_{i}\right\}, i=1,2, \ldots, m,-$ набор точек на ней, а $V$ - голоморфное векторное расслоение ранга $N$ и степени $k$. По аналогии с рациональным случаем определим матрицу Лакса $L^{m|k| N}(z)$ системы Годена $E G(m|k| N)$ на эллиптической кривой $\Sigma$ как мероморфное сечение расслоения End $V$ с простыми полюсами в точках $\left\{z_{i}\right\}$ и фиксированными вычетами $S^{i} \in s l^{*}(N, \mathbb{C})$. Соответствующая 1-форма $L^{m|k| N}(z) d z$ в конструкции Хитчина [1], [2] является редуцированным полем Хиггса. Выбор векторного расслоения $V$ задает квазипериодические граничные условия на решетке $\langle 1, \tau\rangle$ :

$$
L^{m|k| N}(z+1)=g_{1} L^{m|k| N}(z) g_{1}^{-1}, \quad L^{m|k| N}(z+\tau)=g_{\tau} L^{m|k| N}(z) g_{\tau}^{-1} .
$$

По существу, различаются расслоения разных степеней. Размерность пространства модулей расслоений фиксированной степени, ассоциированных с главным $S L(N, \mathbb{C})$ расслоением, равна НОД $(N, k)-1$ [3]. Она максимальна для $k=0 \quad(\bmod N)$ и минимальна для $k=1(\bmod N)$. Эти два случая рассмотрим подробно.

*Институт теоретической и экспериментальной физики, Москва, Россия.

E-mail: zotov@itep.ru

${ }^{\dagger}$ Институт океанологии им. П. П. Ширшова РАН, Москва, Россия.

E-mail: alevin@wave.sio.rssi.ru 
Случай $\operatorname{deg} V=0$ был впервые рассмотрен в работе [4]. Расслоения определяются $N$ параметрами $\left\{u_{i}\right\}, i=1,2, \ldots, N: u_{1}+\cdots+u_{N}=0$. Мультипликаторы заданы в виде

$$
g_{1}=\operatorname{Id}_{N}, \quad g_{\tau}=\mathbf{e}(-\mathbf{u})=\operatorname{diag}\left(\mathbf{e}\left(-u_{1}\right), \ldots, \mathbf{e}\left(-u_{N}\right)\right),
$$

где $\mathbf{e}(x)=e^{2 \pi \sqrt{-1} x}$. Эти условия фиксируют матрицу Лакса системы $E G(m|0| N)$

$$
L_{i j}^{m|0| N}(z)=\delta_{i j} v_{i}+\delta_{i j} \sum_{l=1}^{m} S_{i i}^{l} E_{1}\left(z-z_{l}\right)+\left(1-\delta_{i j}\right) \sum_{l=1}^{m} S_{i j}^{l} \phi\left(z-z_{l}, u_{i}-u_{j}\right)
$$

с точностью до сопряжения произвольным элементом из подгруппы Картана Н в $S L(N, \mathbb{C})$. Функции $E_{1}(z)$ и $\phi(x, y)$ определены в (П.2) и (П.3). Гамильтонова редукция прямого произведения орбит коприсоединенного действия $\left\{\mathcal{O}^{1} \times \cdots \times \mathcal{O}^{m}\right\} / / \mathrm{H}$ по этой симметрии описывает "спиновую" часть фазового пространства системы Годена [5]. Отображение момента, соответствующее указанному действию, имеет вид

$$
\mu=\sum_{l=1}^{m} S_{i i}^{l}
$$

Уравнение $\mu=0$ вместе с некоторым выбором фиксации действия подгруппой $\mathrm{H}$ окончательно задает $L^{m|0| N}(z)$. Бесспиновая часть фазового пространства, соответствующая динамике $N$ частиц в системе центра масс с импульсами $v_{i}$ и координатами $u_{i}$, является кокасательным расслоением к пространству модулей голоморфных векторных расслоений степени ноль.

Отметим важный частный случай: система Годена с одной отмеченной точкой, которой соответствует орбита коприсоединенного действия наименьшей размерности, совпадает после описанной выше редукции с эллиптической $s l(N, \mathbb{C})$-системой Калоджеро-Мозера [6]

$$
L_{i j}^{1|0| N}(z)=\delta_{i j} v_{i}+\left(1-\delta_{i j}\right) \sqrt{-1} \nu \phi\left(z, u_{i}-u_{j}\right),
$$

где $\nu$ - константа взаимодействия. Квадратичный гамильтониан имеет вид

$$
H=\sum_{i=1}^{N} \frac{1}{2} v_{i}^{2}+\sum_{i \neq j} \nu^{2} \wp\left(u_{i}-u_{j}\right) .
$$

Случай $\operatorname{deg} V=1$ был рассмотрен в работе [7]. Мультипликаторы расслоения $V$ задаются в виде

$$
g_{1}=Q^{-1}, \quad g_{\tau}=-\mathbf{e}\left(\frac{\tau}{2 N}+\frac{z}{N}\right) \Lambda^{-1},
$$

где $Q$ и $\Lambda$ - матрицы, задающие стандартное представление конечной группы Гейзенберга (см. приложение). Запишем матрицу Лакса в специальном базисе $\left\{E_{\alpha}\right\}$, 
$\alpha=\left(\alpha_{1}, \alpha_{2}\right) \in \mathbb{Z} / N \mathbb{Z} \times \mathbb{Z} / N \mathbb{Z}$, алгебры Ли $\operatorname{sl}(N, \mathbb{C})$ :

$$
\begin{gathered}
L^{m|1| N}(z)=\sum_{l=1}^{m} \sum_{\alpha \neq 0} E_{\alpha} S_{\alpha}^{l} \varphi_{\alpha}\left(z-z_{l}\right), \quad \varphi_{\alpha}(z)=\mathbf{e}\left(z \partial_{\tau} \omega_{\alpha}\right) \phi\left(z, \omega_{\alpha}\right), \\
\omega_{\alpha}=\frac{\alpha_{1}+\alpha_{2} \tau}{N} .
\end{gathered}
$$

Фазовым пространством $E G(1|1| N)$ является прямое произведение орбит $\mathcal{O}^{1} \times \cdots$ $\cdots \times \mathcal{O}^{m}$ :

$$
\left\{S_{\alpha}^{i}, S_{\beta}^{j}\right\}=2 \sqrt{-1} \sin \left[\frac{\pi}{N}\left(\alpha_{2} \beta_{1}-\alpha_{1} \beta_{2}\right)\right] \delta^{i j} S_{\alpha+\beta}^{i} .
$$

Скобки Пуассона (8) можно задать в $R$-матричной форме

$$
\left\{L_{1}^{m|1| N}(z), L_{2}^{m|1| N}(w)\right\}=\left[L_{1}^{m|1| N}(z)+L_{2}^{m|1| N}(w), r(z, w)\right]
$$

c $R$-матрицей Белавина-Дринфельда [8]

$$
r(z, w)=\sum_{\alpha \neq 0} E_{\alpha} \otimes E_{-\alpha} \varphi_{\alpha}(z-w) .
$$

Соответствие между системой Калоджеро-Мозера и эллиптическим волчком. Модификация была определена в [9] как процедура, изменяющая степень векторного расслоения $V$ на единицу. На сечениях $\operatorname{End} V$ эта процедура действует калибровочным преобразованием, вырожденным в фиксированной точке. В работах [10] было построено преобразование, связывающее $L^{1|0| N}$ и $L^{1|1| N}$, т.е. установлена калибровочная эквивалентность между эллиптической системой Калоджеро-Мозера и эллиптическим волчком. В случае $\operatorname{sl}(2, \mathbb{C})$ нетрудно получить явную замену переменных:

$$
\begin{gathered}
\{v, u\}=1, \quad\left\{S_{\alpha}, S_{\beta}\right\}=2 \sqrt{-1} \varepsilon_{\alpha \beta \gamma} S_{\gamma} \\
L^{1|0| 2}=\left(\begin{array}{cc}
v & \nu \phi(2 u, z) \\
\nu \phi(-2 u, z) & -v
\end{array}\right) \\
L^{1|1| 2}=\left(\begin{array}{cc}
S_{3} \varphi_{3}(z) & S_{1} \varphi_{1}(z)-i S_{2} \varphi_{2}(z) \\
S_{1} \varphi_{1}(z)+i S_{2} \varphi_{2}(z) & -S_{3} \varphi_{3}(z)
\end{array}\right),
\end{gathered}
$$

где индексы согласованы с нумерацией матриц Паули: $(1,2,3)=(01,11,01)$. Утверждение об эквивалентности двух систем означает, что существует калибровочное преобразование $\Xi(z)$ такое, что

$$
L^{1|1| 2}(z)=\Xi(z) L^{1|0| 2}(z) \Xi^{-1}(z) .
$$

Как показано в $[10]$, в случае $s l(2, \mathbb{C})$ преобразование имеет вид

$$
\Xi(z)=\left(\begin{array}{cc}
\theta_{00}(z-2 u, 2 \tau) & -\theta_{00}(z+2 u, 2 \tau) \\
-\theta_{10}(z-2 u, 2 \tau) & \theta_{10}(z+2 u, 2 \tau)
\end{array}\right) .
$$


Тогда из (12) следует, что

$$
\begin{aligned}
S_{01} & =-v \frac{\theta_{01}(0)}{\vartheta^{\prime}(0)} \frac{\theta_{01}(2 u)}{\vartheta(2 u)}+\nu \frac{\theta_{01}^{2}(0)}{\theta_{00}(0) \theta_{10}(0)} \frac{\theta_{00}(2 u) \theta_{10}(2 u)}{\vartheta^{2}(2 u)}, \\
-\sqrt{-1} S_{11} & =-v \frac{\theta_{00}(0)}{\vartheta^{\prime}(0)} \frac{\theta_{00}(2 u)}{\vartheta(2 u)}+\nu \frac{\theta_{00}^{2}(0)}{\theta_{10}(0) \theta_{01}(0)} \frac{\theta_{10}(2 u) \theta_{01}(2 u)}{\vartheta^{2}(2 u)}, \\
S_{10} & =-v \frac{\theta_{10}(0)}{\vartheta^{\prime}(0)} \frac{\theta_{10}(2 u)}{\vartheta(2 u)}+\nu \frac{\theta_{10}^{2}(0)}{\theta_{00}(0) \theta_{01}(0)} \frac{\theta_{00}(2 u) \theta_{01}(2 u)}{\vartheta^{2}(2 u)} .
\end{aligned}
$$

\section{2. СИСТЕМА ВЗАИМОДЕЙСТВУЮЩИХ ВОЛЧКОВ}

Рассмотрим эллиптический волчок на расслоении степени $n$ и ранга $N=n p$, $N>n$. Это означает, что квазипериодические граничные условия для матрицы Лакса выглядят следующим образом:

$$
\begin{aligned}
& L(z+1)=Q L(z) Q^{-1}, \\
& L(z+\tau)=\Lambda^{n} L(z) \Lambda^{-n} .
\end{aligned}
$$

Однако таким условиям может удовлетворять только вырожденная матрица $L(z)$. Дело в том, что существует диагональная матрица, содержащая $n$ различных собственных значений, $\bar{A}=\operatorname{diag}\left\{u_{1}, \ldots, u_{n}, p, u_{1}, \ldots, u_{n}\right\}$, которая коммутирует одновременно с $Q$ и $\Lambda^{n}$ :

$$
Q \bar{A}=\bar{A} Q, \quad \Lambda^{n} \bar{A}=\bar{A} \Lambda^{n} .
$$

Чтобы зафиксировать эту свободу, модифицируем условия (15):

$$
\begin{aligned}
& L(z+1)=Q L(z) Q^{-1}, \\
& L(z+\tau)=\mathbf{e}(-\bar{A}) \Lambda^{n} L(z) \Lambda^{-n} \mathbf{e}(\bar{A}) .
\end{aligned}
$$

Эти граничные условия отвечают существованию $(n-1)$-мерного пространства модулей.

УтвеРжДЕниЕ. Существует такая числовая матрица $M$, что

$$
\begin{aligned}
M \bar{A} M^{-1} & =\bigoplus_{J=1}^{n} u_{J} \operatorname{Id}_{p \times p}, \\
M Q M^{-1} & =\bigoplus_{J=1}^{n} \mathbf{e}\left(\frac{J-p}{N}\right) Q_{p \times p}, \\
M \Lambda^{n} M^{-1} & =\bigoplus_{J=1}^{n} \Lambda_{p \times p} .
\end{aligned}
$$

Последнее равенство означает, что $\Lambda^{n}$ приводится к блочно-диагональному виду, причем количество блоков равно $n$, и каждый блок представляет из себя матрицу $\Lambda$ размером $p \times p$. 
Доказательство. Пусть $m=(\alpha-1) n+\beta$, где $\alpha=1,2, \ldots, p, \beta=1,2, \ldots, n$. Определим операцию перестановки

$$
\psi_{n, p}(m)=(\beta-1) p+\alpha
$$

Наше утверждение состоит в том, что искомая матрица имеет вид

$$
M_{i j}=\delta\left(\psi_{p, n}(i), j\right)
$$

Проверим это. Заметим, что для обратной матрицы верно

$$
\left(M^{-1}\right)_{k l}=\delta\left(\psi_{n, p}(k), l\right) .
$$

Итак,

$$
M_{i j} \bar{A}_{j k} M_{k l}^{-1}=\delta\left(\psi_{p, n}(i), j\right) \delta(j, k) \bar{A}_{j j} \delta\left(\psi_{n, p}(k), l\right)=\delta\left(\psi_{n, p}(i), l\right) \bar{A}_{i i}
$$

(по повторяющимся индексам предполагаются свертки). Далее, имеем

$$
\begin{aligned}
M_{i k} Q_{k l} M_{l i}^{-1} & =\delta(i, j) \mathbf{e}\left(\frac{\psi_{p, n}(i)}{N}\right) \\
M_{i j} \Lambda_{j k}^{n} M_{k l}^{-1} & =\delta\left(\psi_{p, n}(i), j\right) \delta\left(\bmod _{N}(j+n), k\right) \delta\left(\psi_{n, p}(k), l\right)= \\
& =\delta\left(\psi_{n, p}\left(\bmod _{N}\left(\psi_{p, n}(i)+n\right)\right), l\right) .
\end{aligned}
$$

Пусть теперь $i=(\beta-1) p+\alpha$, тогда:

а) если $\alpha<p$, то $\bmod _{N}\left(\psi_{p, n}(i)+n\right)=\psi_{p, n}(i)+n=\alpha n+\beta$, а $\psi_{n, p}(\alpha n+\beta)=$ $(\beta-1) p+\alpha+1=i+1$

б) если $\alpha=p$, то $\bmod _{N}((p-1) n+\beta+n)=\beta$, а $\psi_{n, p}(\beta)=(\beta-1) p+1$.

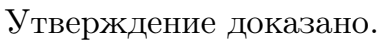

В дальнейшем мы будем использовать прописные буквы латинского алфавита для индекса, пробегающего от 1 до $n$, а строчные буквы - для индекса, пробегающего от 1 до $p$. Кроме того, примем обозначение $\sum_{m, n}=\sum_{m, n=0, m^{2}+n^{2} \neq 0}^{p-1}$.

Воспользуемся доказанным утверждением и перейдем к твистованному базису. Тогда для блоков размера $p \times p$ имеем

$$
\begin{aligned}
& L_{I J}(z+1)=\mathbf{e}\left(\frac{I-J}{N}\right) Q_{p \times p} L_{I J}(z) Q_{p \times p}^{-1}, \\
& L_{I J}(z+\tau)=\mathbf{e}\left(-u_{I}\right) \Lambda_{p \times p} L_{I J}(z) \Lambda_{p \times p}^{-1} \mathbf{e}\left(u_{J}\right) .
\end{aligned}
$$

Множитель $\mathbf{e}((I-J) / N)$ устраняется заменой

$$
L_{I J}(z) \rightarrow L_{I J}(z) \mathbf{e}\left(-z \frac{I-J}{N}\right), \quad u_{I} \rightarrow u_{I}-I \frac{\tau}{N} .
$$

В итоге получаем следующую форму граничных условий:

$$
\begin{aligned}
& L_{I J}(z+1)=Q_{p \times p} L_{I J}(z) Q_{p \times p}^{-1}, \\
& L_{I J}(z+\tau)=\mathbf{e}\left(-u_{I}\right) \Lambda_{p \times p} L_{I J}(z) \Lambda_{p \times p}^{-1} \mathbf{e}\left(u_{J}\right) .
\end{aligned}
$$


Оператор $L$, удовлетворяющий условиям (19) и имеющий фиксированный вычет, можно легко получить:

$$
\begin{gathered}
L_{I J}(z)=\frac{1}{p} \delta_{I J} v_{I}+\sum_{m, n}\left(S_{I J}\right)_{m n} \phi_{m n}\left(z, u_{I J}\right) E_{m n} \\
\phi_{m n}\left(z, u_{I J}\right)=\mathbf{e}\left(\frac{-n z}{N}\right) \phi\left(u_{I J}-\frac{m+n \tau}{N}, z\right) .
\end{gathered}
$$

Множитель $1 / p$ поставлен перед импульсом для того, чтобы сохранить канонической скобку Пуассона $\left\{v_{I}, u_{J}\right\}=\delta_{I J}$.

Скобки Пуассона для матричных элементов $S$ задаются в виде скобок ПуассонаЛи, построенных по структурным константам алгебры Ли $g l(N, \mathbb{C})$ :

$$
\left\{\left(S_{I J}\right)_{a b},\left(S_{K L}\right)_{c d}\right\}=2 \sqrt{-1} \sin \left[\frac{\pi}{p}(b c-a d)\right]\left(\delta_{K J}\left(S_{I L}\right)_{a+c, b+d}-\delta_{I L}\left(S_{K J}\right)_{a+c, b+d}\right) .
$$

Здесь использованы два различных базиса: стандартный для блоков размера $p \times p$ $(I, J=1,2, \ldots, n)$ и базис синус-алгебры (П.13)-(П.16) для элементов блоков.

Квадратичный гамильтониан имеет вид

$$
H=\frac{1}{2} \sum_{I=1}^{n} v_{I}^{2}-\frac{1}{2} \sum_{I, J} \sum_{m, n} \operatorname{Tr}\left(S_{I J} E_{-m,-n}\right) \operatorname{Tr}\left(S_{J I} E_{m n}\right) E_{2}\left(u_{I J}-\frac{m+n \tau}{N}\right),
$$

или

$$
\begin{aligned}
H= & \frac{1}{2} \sum_{I=1}^{n} v_{I}^{2}-\frac{1}{2} \sum_{I} \sum_{m, n} \operatorname{Tr}\left(S_{I I} E_{-m,-n}\right) \operatorname{Tr}\left(S_{I I} E_{m n}\right) E_{2}\left(\frac{m+n \tau}{N}\right)- \\
& -\frac{1}{2} \sum_{I \neq J} \sum_{m, n} \operatorname{Tr}\left(S_{I J} E_{-m,-n}\right) \operatorname{Tr}\left(S_{J I} E_{m n}\right) E_{2}\left(u_{I J}-\frac{m+n \tau}{N}\right) .
\end{aligned}
$$

Первые два слагаемых представляют собой гамильтонианы $n(p \times p)$-волчков с импульсами $v_{I}$. Заметим, что аналог редукции по подгруппе Картана при $\operatorname{deg} V=0$ в данном случае приводит к требованию $\operatorname{Tr}\left(S_{I I}\right)=$ const $\forall I$. Хотелось бы интерпретировать последнее слагаемое как потенциал их взаимодействия. Это можно сделать, если $S$ принадлежит орбите наименьшей размерности, т.е. $r k S=1$. Действительно, воспользуемся известной параметризацией [11] такой орбиты. Положим для простоты, что $S \in g l(N, \mathbb{C})$, тогда $S=\xi \times \eta$, где $\xi$ и $\eta$ - столбец и строка длины $N$, причем $\left\{\xi_{a}, \eta_{b}\right\}=\delta_{a b}$. Следовательно,

$$
\operatorname{Tr}\left(S_{I J} E_{-m,-n}\right) \operatorname{Tr}\left(S_{J I} E_{m n}\right)=\operatorname{Tr}\left(S_{I I} E_{m n} S_{J J} E_{-m,-n}\right)
$$

Условие $\operatorname{Tr}\left(S_{I I}\right)=$ const означает, что матрицы $S_{I I}$ описывают орбиты коприсоединенного представления наименьшей размерности, равной $2 p-2$. В этом случае 
гамильтониан запишется в виде, имеющем более прозрачную физическую интерпретацию:

$$
\begin{aligned}
H= & \frac{1}{2} \sum_{I=1}^{n} v_{I}^{2}-\frac{1}{2} \sum_{I} \sum_{m, n} \operatorname{Tr}\left(S_{I I} E_{-m,-n}\right) \operatorname{Tr}\left(S_{I I} E_{m n}\right) E_{2}\left(\frac{m+n \tau}{N}\right)- \\
& -\frac{1}{2} \sum_{I \neq J} \sum_{m, n} \operatorname{Tr}\left(S_{I I} E_{m, n} S_{J J} E_{-m,-n}\right) E_{2}\left(u_{I J}-\frac{m+n \tau}{N}\right) .
\end{aligned}
$$

В этой формуле первые два члена описывают энергию $n(p \times p)$-волчков, а последний - попарное взаимодействие между ними. Как было показано в [10], все системы из семейства $E G(1|k| N), \quad k=1,2, \ldots, N$, симплектоморфны. Тем самым построенная система взаимодействующих волчков симплектоморфна, например, $N$ частичной системе Калоджеро-Мозера.

\section{ПРИЛОЖКНИЕ 1}

\section{Эллиптические функции}

В этом приложении изложены основные определения и соотношения для эллиптических функций, необходимые для проверки результатов, представленных в данной работе. Большинство формул заимствованы из [12] и [13].

Введем обозначение $q=e^{2 \pi i \tau}$, где $\tau$ - модулярный параметр эллиптической кривой $E_{\tau}$.

Основной объект - тета-функция:

$$
\begin{aligned}
\vartheta(z \mid \tau) & =q^{1 / 8} \sum_{n \in \mathbb{Z}}(-1)^{n} e^{\pi i(n(n+1) \tau+2 n z)}= \\
& =q^{1 / 8} e^{-i \pi / 4}\left(e^{i \pi z}-e^{-i \pi z}\right) \prod_{n=1}^{\infty}\left(1-q^{n}\right)\left(1-q^{n} e^{2 i \pi z}\right)\left(1-q^{n} e^{-2 i \pi z}\right) .
\end{aligned}
$$

Функции Эйзенштейна:

$$
E_{1}(z \mid \tau)=\partial_{z} \ln \vartheta(z \mid \tau), \quad E_{1}(z \mid \tau) \sim \frac{1}{z}-2 \eta_{1} z
$$

где

$$
\eta_{1}(\tau)=\zeta\left(\frac{1}{2}\right)=\frac{3}{\pi^{2}} \sum_{m=-\infty}^{\infty} \sum_{n=-\infty}^{\infty^{\prime}} \frac{1}{(m \tau+n)^{2}}=\frac{24}{2 \pi i} \frac{\eta^{\prime}(\tau)}{\eta(\tau)}
$$

где

$$
\eta(\tau)=q^{1 / 24} \prod_{n>0}\left(1-q^{n}\right)
$$

- функция Дедекинда.

Второй ряд Эйзенштейна имеет вид

$$
E_{2}(z \mid \tau)=-\partial_{z} E_{1}(z \mid \tau)=\partial_{z}^{2} \ln \vartheta(z \mid \tau), \quad E_{2}(z \mid \tau) \sim \frac{1}{z^{2}}+2 \eta_{1}
$$


Следующий важный объект:

$$
\phi(u, z)=\frac{\vartheta(u+z) \vartheta^{\prime}(0)}{\vartheta(u) \vartheta(z)}, \quad \varphi_{\alpha}(z, \alpha+u)=\mathbf{e}\left(z \partial_{\tau} \alpha\right) \phi(z, \alpha+u) .
$$

Эта функция имеет полюс в точке $z=0$ и разлагается в ее окрестности в следующий ряд:

$$
\phi(u, z)=\frac{1}{z}+E_{1}(u)+\frac{z}{2}\left(E_{1}^{2}(u)-\wp(u)\right)+\cdots .
$$

Формула для производной:

$$
\phi(u, z)^{-1} \partial_{u} \phi(u, z)=E_{1}(u+z)-E_{1}(u) .
$$

Связь с функциями Вейерштрасса:

$$
\begin{gathered}
\zeta(z \mid \tau)=E_{1}(z \mid \tau)+2 \eta_{1}(\tau) z, \quad \wp(z \mid \tau)=E_{2}(z \mid \tau)-2 \eta_{1}(\tau), \\
\phi(u, z)=e^{-2 \eta_{1} u z} \frac{\sigma(u+z)}{\sigma(u) \sigma(z)} \\
\phi(u, z) \phi(-u, z)=\wp(z)-\wp(u)=E_{2}(z)-E_{2}(u) .
\end{gathered}
$$

Представление в виде рядов:

$$
\begin{aligned}
E_{1}(z \mid \tau) & =-2 \pi i\left(\frac{1}{2}+\sum_{n \neq 0} \frac{e^{2 \pi i z}}{1-q^{n}}\right)= \\
& =-2 \pi i\left(\sum_{n<0} \frac{1}{1-q^{n} e^{2 \pi i z}}+\sum_{n \geqslant 0} \frac{q^{n} e^{2 \pi i z}}{1-q^{n} e^{2 \pi i z}}+\frac{1}{2}\right), \\
E_{2}(z \mid \tau) & =-4 \pi^{2} \sum_{n \in \mathbb{Z}} \frac{q^{n} e^{2 \pi i z}}{\left(1-q^{n} e^{2 \pi i z}\right)^{2}}, \\
\phi(u, z) & =2 \pi i \sum_{n \in \mathbb{Z}} \frac{e^{-2 \pi i n z}}{1-q^{n} e^{-2 \pi i u}} .
\end{aligned}
$$

Четность:

$$
\begin{aligned}
& \vartheta(-z)=-\vartheta(z), \quad E_{1}(-z)=-E_{1}(z), \quad E_{2}(-z)=E_{2}(z), \\
& \phi(u, z)=\phi(z, u)=-\phi(-u,-z) .
\end{aligned}
$$

Квазипериодические свойства:

$$
\begin{gathered}
\vartheta(z+1)=-\vartheta(z), \quad \vartheta(z+\tau)=-q^{-1 / 2} e^{-2 \pi \sqrt{-1} z} \vartheta(z), \\
E_{1}\left(z+2 \omega_{\alpha}\right)=E_{1}(z)-4 \pi \sqrt{-1} \partial_{\tau} \omega_{\alpha}, \\
E_{1}(z+1)=E_{1}(z), \quad E_{1}(z+\tau)=E_{1}(z)-2 \pi \sqrt{-1}, \\
E_{2}\left(z+2 \omega_{\alpha}\right)=E_{2}(z), \quad E_{2}(z+1)=E_{2}(z), \quad E_{2}(z+\tau)=E_{2}(z), \\
\phi(u+1, z)=\phi(z, u), \quad \phi(u+\tau, z)=e^{-2 \pi \sqrt{-1} z} \phi(z, u) .
\end{gathered}
$$


Формулы сложения:

$$
\phi(u, z) \partial_{v} \phi(v, z)-\phi(v, z) \partial_{u} \phi(u, z)=\left(E_{2}(v)-E_{2}(u)\right) \phi(u+v, z)
$$

или

$$
\phi(u, z) \partial_{v} \phi(v, z)-\phi(v, z) \partial_{u} \phi(u, z)=(\wp(v)-\wp(u)) \phi(u+v, z) .
$$

Доказательство формулы (П.9) основано на использовании (П.4) и формул (П.7), (П.8) для функции $\phi(u, z)$.

В действительности функция $\phi(u, z)$ удовлетворяет более общему соотношению, следующему из тождества Фэя для тройной секущей:

$$
\phi\left(u_{1}, z_{1}\right) \phi\left(u_{2}, z_{2}\right)-\phi\left(u_{1}+u_{2}, z_{1}\right) \phi\left(u_{2}, z_{2}-z_{1}\right)-\phi\left(u_{1}+u_{2}, z_{2}\right) \phi\left(u_{1}, z_{1}-z_{2}\right)=0 .
$$

Частным случаем этой формулы является соотношение

$$
\phi\left(u_{1}, z\right) \phi\left(u_{2}, z\right)-\phi\left(u_{1}+u_{2}, z\right)\left(E_{1}\left(u_{1}\right)+E_{1}\left(u_{2}\right)\right)+\partial_{z} \phi\left(u_{1}+u_{2}, z\right)=0 .
$$

ПРИЛОЖЕНИЕ 2

\section{Синус-алгебра}

Генераторы синус-алгебры $E_{m n}$ определяются через генераторы конечной группы Гейзенберга $Q$ и $\Lambda$ :

$$
\begin{aligned}
E_{m n} & =\mathbf{e}\left(\frac{m n}{2 N}\right) Q^{m} \Lambda^{n}, \quad m=0,1, \ldots, N-1, \\
n & =0,1, \ldots, N-1, \quad m^{2}+n^{2} \neq 0 \quad(\bmod N),
\end{aligned}
$$

в базисе $\operatorname{sl}(N, \mathbb{C})$, где

$$
\begin{gathered}
\mathbf{e}(z)=e^{2 \pi \sqrt{-1} z}, \quad Q=\operatorname{diag}(\mathbf{e}(1 / N), \ldots, \mathbf{e}(m / N), \ldots, 1), \\
\Lambda=\left(\begin{array}{ccccc}
0 & 1 & 0 & \ldots & 0 \\
0 & 0 & 1 & \ldots & 0 \\
\vdots & \vdots & \vdots & \ddots & \vdots \\
0 & 0 & 0 & \ldots & 1 \\
1 & 0 & 0 & \ldots & 0
\end{array}\right)
\end{gathered}
$$

Коммутационные соотношения имеют вид

$$
\begin{aligned}
{\left[E_{s k}, E_{n j}\right] } & =2 \sqrt{-1} \sin \left[\frac{\pi}{N}(k n-s j)\right] E_{s+n, k+j}, \\
\operatorname{Tr}\left(E_{s k} E_{n j}\right) & =\delta_{s,-n} \delta_{k,-j} N .
\end{aligned}
$$


Благодарности. Авторы выражают благодарность за поддержку РФФИ (грант № 03-02-17554), а также Программе поддержки научных школ (грант № НШ1999.2003.2) и CRDF (грант № RM1-2545-MO-03).

\section{Список литературы}

[1] N. Hitchin. Duke Math. J. 1987. V. 54. P. 91-114.

[2] A. Levin, M. Olshanetsky. Commun. Math. Phys. 1997. V. 188. P. 449-466.

[3] M. F. Atiyah. Proc. London Math. Soc. 1957. V. 7. P. 414-452.

[4] N. Nekrasov. Commun. Math. Phys. 1996. V. 180. P. 587-604; hep-th/9503157.

[5] I. Krichever, O. Babelon, E. Billey, M. Talon. Amer. Math. Soc. Transl. 1995. V. 170. P. 83-119.

[6] F. Calogero. J. Math. Phys. 1971. V. 12. P. 419-436; Erratum. 1996. V. 37. P. 4636; J. Moser. Adv. Math. 1975. V. 16. P. 197-220.

[7] А. Г. Рейман, М. А. Семенов-Тян-Шанский. Зап. научн. семин. ЛОМИ. 1986. Т. 150. C. $104-118$.

[8] A. A. Belavin. Nucl. Phys. 1981. V. 180 [FS2]. P. 189-200.

[9] D. Arinkin, S. Lysenko. Math. Res. Lett. 1997. V. 4. P. 181-190.

[10] A. Levin, M. Olshanetsky, A. Zotov. Commun. Math. Phys. 2003. V. 236. P. 93-133; Math. Ann. 1980. V. 248. P. 205-239.

[11] J. Avan, O. Babelon, M. Talon. Алгебра и анализ. 1994. Т. 6. № 2. С. 67-89; hep-th/9306102.

[12] А. Вейль. Эллиптические функции по Эйзенштейну и Кронекеру. М.: Мир, 1978.

[13] Д. Мамфорд. Лекции о тэта-функциях. М.: Мир, 1988. 